\title{
Endoscopic Submucosal Dissection of an Inverted Early Gastric Cancer-Forming False Gastric Diverticulum
}

\author{
Yong-il Lee and Sang-kil Lee \\ Division of Gastroenterology, Department of Internal Medicine, Yonsei University College of Medicine, Seoul, Korea
}

Endoscopic submucosal dissection (ESD) is a standard treatment for early gastric cancer (EGC) that does not have any risk of lymph node or distant metastases. Here, we report a case of EGC resembling a diverticulum. Diverticular formation makes it difficult for endoscopists to determine the depth of invasion and to subsequently perform ESD. Because the false diverticulum does not have a muscular layer, this lesion can be treated with ESD. Our case was successfully treated with ESD. After ESD, the EGC was confined to the submucosal layer without vertical and lateral margin involvement. This is the first case in which ESD was successfully performed for a case of EGC that coexisted with a false gastric diverticulum. An additional, larger study is needed to determine the efficacy of ESD in various types of EGC, such as a false gastric diverticulum. Clin Endosc 2016;49:86-90

Key Words: Stomach neoplasms; Endoscopy, digestive system; Diverticulum, stomach

\section{INTRODUCTION}

Endoscopic submucosal dissection (ESD) is a standard treatment for early gastric cancer (EGC) that does not have any risk of lymph node or distant metastases. The indication for ESD is determined by the lesion size, shape, ulceration, depth of invasion, and histologic differentiation. Here, we report a case of EGC that resembled a diverticulum, which made it difficult to estimate the lesion size, shape, ulceration, and depth of invasion. This lesion was treated with ESD, which revealed type I+IIc EGC confined to the submucosal layer, shaped like a false diverticulum because of submucosal inflammation and infiltration.

\footnotetext{
Received: March 30, 2015 Revised: June 1, 2015

Accepted: June 2, 2015

Correspondence: Sang-kil Lee

Division of Gastroenterology, Department of Internal Medicine, Yonsei University College of Medicine, 50-1 Yonsei-ro, Seodaemun-gu, Seoul 03722, Korea

Tel: +82-2-2228-1996, Fax: +82-2-393-6884, E-mail: sklee@yuhs.ac

(cc) This is an Open Access article distributed under the terms of the Creative Commons Attribution Non-Commercial License (http://creativecommons.org/ licenses/by-nc/3.0) which permits unrestricted non-commercial use, distribution, and reproduction in any medium, provided the original work is properly cited.
}

\section{CASE REPORT}

A 47-year-old Korean man visited our hospital for periodic health examination in October 2014. He had a history of immunoglobulin G nephropathy, and had undergone bilateral kidney transplantation 15 years ago. He had an intraductal papillary mucinous neoplasm of the pancreas, and underwent a distal pancreatectomy and splenectomy in October 2012. All laboratory findings were within the reference limits. We performed an esophagogastroduodenoscopy (EGD), endoscopic ultrasound (EUS), and abdomen-pelvis computed tomography (CT). On EGD, the EGC was located in the lesser curvature of the antrum. The EGC was shaped like an elevated nodular mucosal lesion with a central inverted area. The maximal longitudinal size of the lesion was $10 \mathrm{~mm}$. The operation history may have contributed to the shape and the inverted type of the central depressed lesion, such as a false diverticulum. This false diverticulum overlaid the superficial elevated EGC in the submucosal layer. The EGC was $15 \mathrm{~mm}$ in size and classified as type I+IIc according to the Japanese classification. ${ }^{1}$ The false diverticulum showed spontaneous bleeding and a reddish surface (Fig. 1A). Endoscopic forceps biopsy was performed three times at the anterior wall, two times at the posterior wall, and three times at the center 

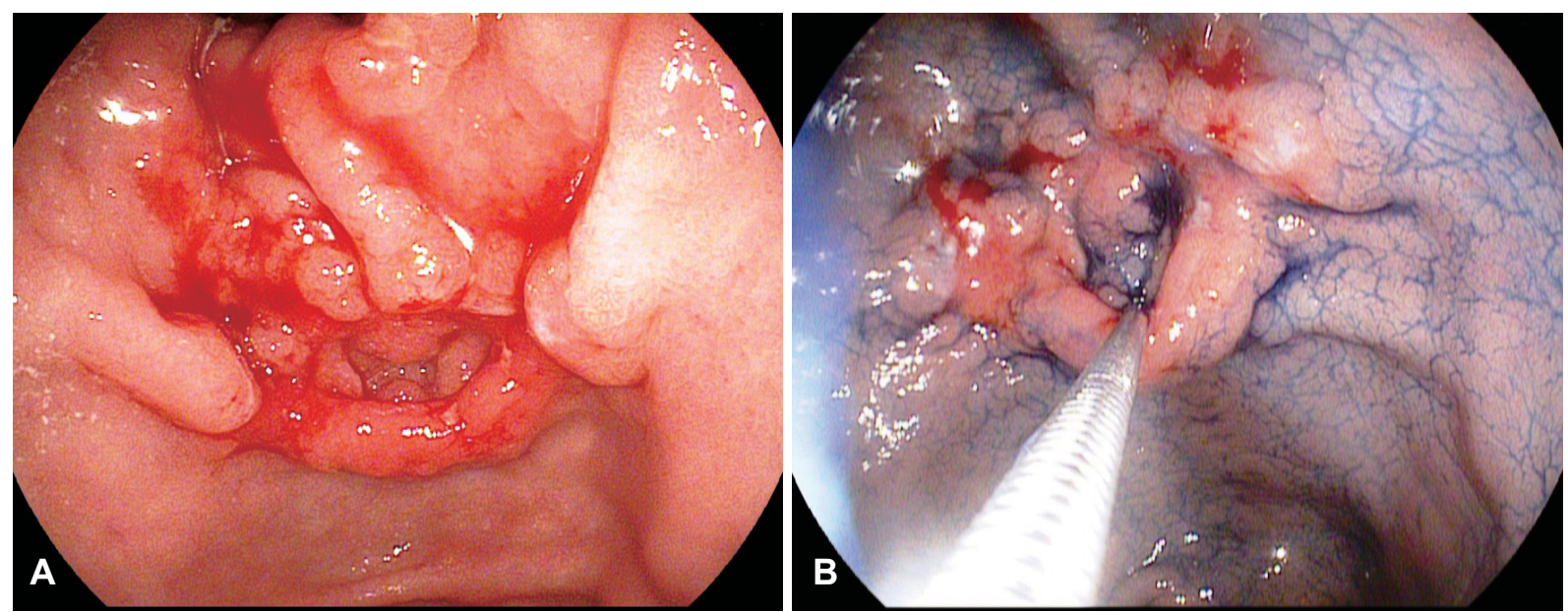

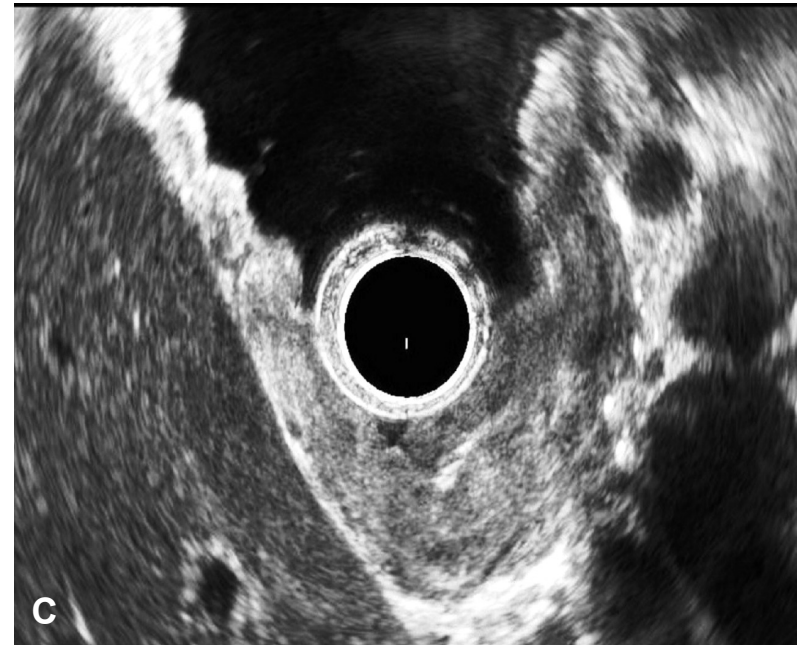

opening of the main lesion. The pathology analysis revealed a moderate, well-differentiated tubular adenocarcinoma (Fig. 1B). The EUS showed that the main lesion was mostly located in the mucosal layer; however, partial submucosal invasion was observed, and there was no regional lymph node enlargement (Fig. 1C). On CT scan, there were no other stomach lesions and no lymph enlargement.

Because of his prior surgery, he needed a total gastrectomy although the lesion was located on the antrum. However, both he and the surgeon were reluctant about surgery. For this reason, although the exact character of the lesion was uncertain, ESD was done to resect the EGC.

For the ESD technique, we used a GIF-Q260J instrument (Olympus Optical Co. Ltd., Tokyo, Japan). First, circumferential markings were created around the lesion with argon plasma coagulation. The lesion was raised by means of a submucosal injection of a $37 \mathrm{~mL}$ mixture of indigo carmine, epinephrine, and hypertonic saline $(1: 100,000)$. Although it was difficult to differentiate between true and false diverticulum in this lesion, lifting of the lesion with indigo carmine and epinephrine mixed in saline would suggest that the lesion
Fig. 1. Esophagogastroduodenoscopy and endoscopic ultrasound. (A) False gastric diverticulum coexisting with type I+Ilc early gastric cancer with a central opening. Spontaneous bleeding and a reddish surface are evident. (B) Forceps biopsies performed three times at the anterior wall, two times at the posterior wall, and three times at the central opening of the main lesion. (C) The invasion of the submucosal layer is unclear because of a previous operation, and no lymph node invasion is observed.

is a false diverticulum induced by inflammation or the previous operation. After the injection of indigo carmine solution, the submucosal layer appeared grossly as a white web-like structure (Fig. 2A). Precutting incisions were done around the lesion. (Fig. 2B). In addition, full submucosal dissection was done by using an insulation-tipped (IT) knife and a hook knife, with a coagulation current of $60 \mathrm{~W}$ (Fig. 2C, D). The en bloc resected tumor was $5.6 \times 4.7 \mathrm{~cm}$ in size and had a central opening with nodular submucosal exposure (Fig. 2E).

A negative endoscopic gross resection margin was assured after tentative histologic examination. The safety margin was $0.9 \mathrm{~cm}$ of the proximal part, $0.15 \mathrm{~cm}$ of the distal part, $1.0 \mathrm{~cm}$ of the anterior wall, $0.4 \mathrm{~cm}$ of the posterior wall, and $150 \mu \mathrm{m}$ of the basal part. No lymphovascular invasion or perineural invasion was seen. Type I+IIc EGC coexisted with the false diverticulum, and the pathologic tumor size was $3.6 \times 1.8 \mathrm{~cm}$, which was larger than the extended criteria for ESD (Fig. $3 \mathrm{~A}){ }^{2}$ The cancer was a tubular adenocarcinoma, intestinal type, based on the Lauren classification, and was moderately differentiated. Invasion of the submucosa (pTlb, depth of submucosal invasion: $450 \mu \mathrm{m}$ ) was observed (Fig. 3B). 

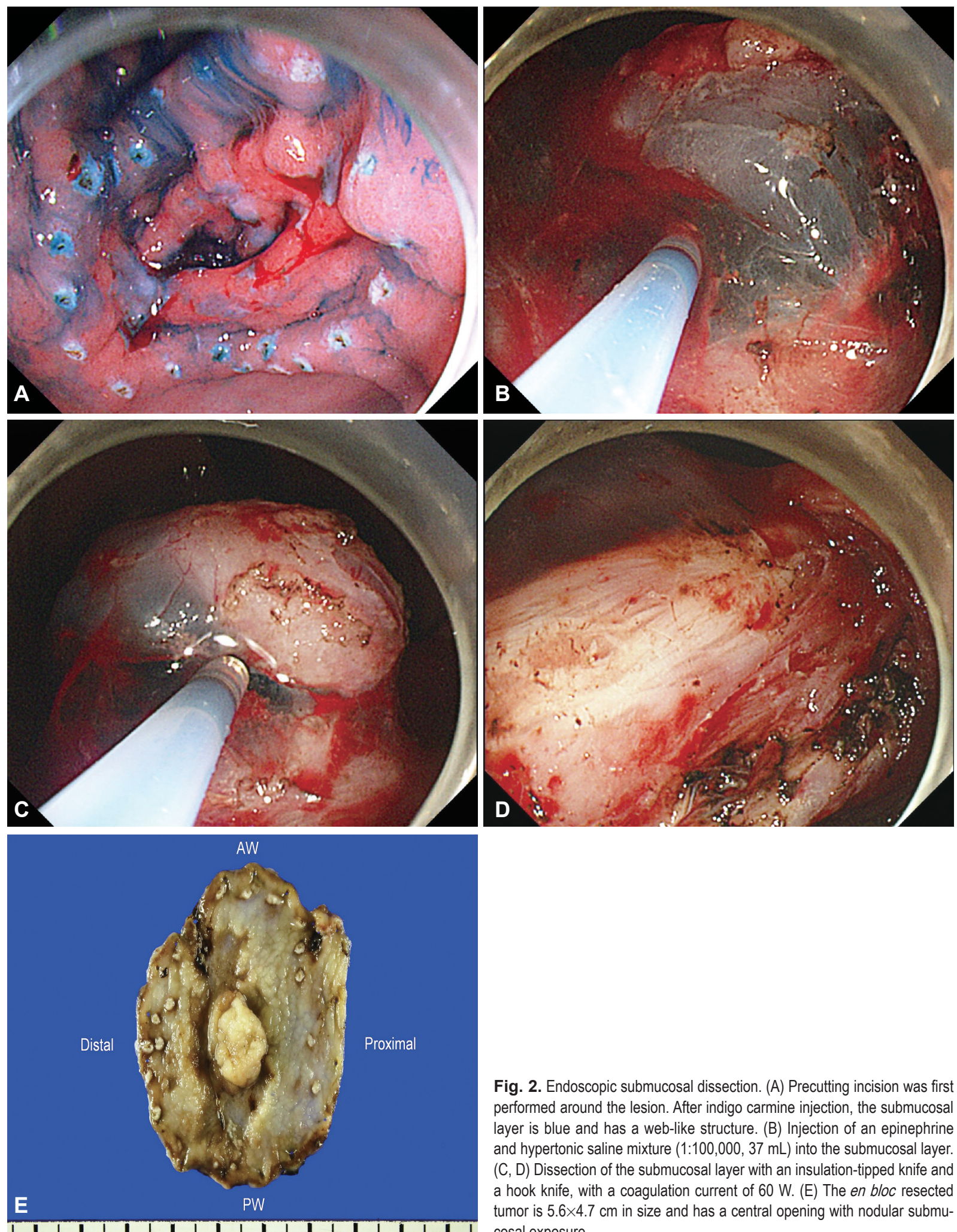

Fig. 2. Endoscopic submucosal dissection. (A) Precutting incision was first performed around the lesion. After indigo carmine injection, the submucosal layer is blue and has a web-like structure. (B) Injection of an epinephrine and hypertonic saline mixture $(1: 100,000,37 \mathrm{~mL})$ into the submucosal layer. (C, D) Dissection of the submucosal layer with an insulation-tipped knife and a hook knife, with a coagulation current of $60 \mathrm{~W}$. (E) The en bloc resected tumor is $5.6 \times 4.7 \mathrm{~cm}$ in size and has a central opening with nodular submucosal exposure.

He had no specific complications and was discharged from go follow-up care instead of surgery. the hospital after 4 days. On the basis of the pathologic report, additional surgery was needed; however, he wanted to under- 

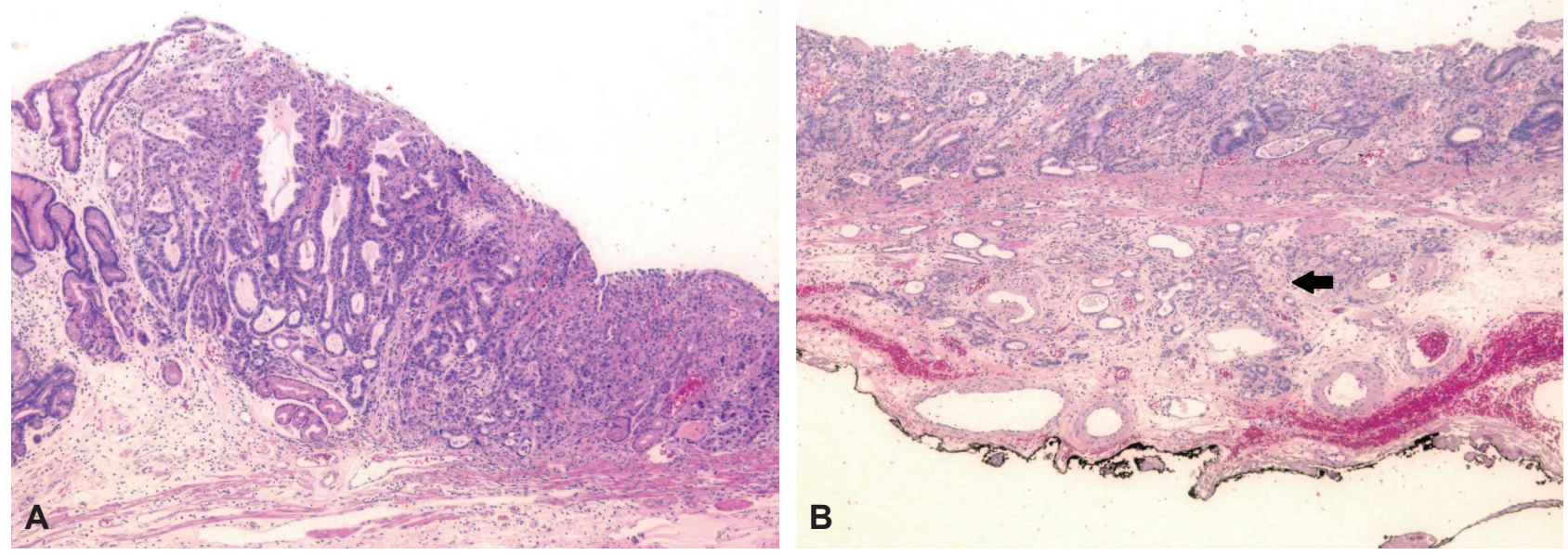

Fig. 3. Histologic features of the resected specimen. (A) The type I+llc early gastric cancer coexisting with a diverticulum is a moderately to well-differentiated tubular adenocarcinoma. The tumor size is $3.6 \times 1.8 \mathrm{~cm}$ (H\&E stain, $\times 40$ ). (B) The tumor invading the submucosal layer (black arrow). The depth of submucosal invasion is $450 \mu \mathrm{m}$. The safety margin of the base is $150 \mu \mathrm{m}$ (black ink; H\&E stain, $\times 40$ ).

\section{DISCUSSION}

ESD was developed for the complete removal of EGC in the late 1990s, by using a scope with endoscopic knives. ${ }^{3}$ In 2007, Gotoda ${ }^{4}$ proposed the extended ESD criteria for EGD. The extended ESD criteria were compatible with surgical resection for EGC in terms of safety, efficacy, risk of recurrence, and complications. Endoscopic resection has been well established as a standard therapy for EGC in Korea. Additionally, the management of EGC has improved owing to advances in ESD applications.

The definition of gastric diverticulum was suggested in 1661 by Moebius in rare cases of gastric wall transformation. ${ }^{5}$ The prevalence of gastric diverticulum ranges from $0.01 \%$ to $0.04 \%$. Recent articles have commented that the best diagnostic tool for detecting a gastric diverticulum is upper gastrointestinal contrast radiography or EGD. Additionally, this lesion is often incidentally detected on $\mathrm{CT}^{6}$.

Two types of gastric diverticulum have been identified. First, true (congenital) diverticulum is relatively common, and comprises $70 \%$ to $75 \%$ of all gastric diverticulum cases. They are usually located on the posterior wall of the cardiac region. On the 20th and 50th days of gestation, the stomach is transformed from a fusiform swelling of the foregut into its adult form, during which a $90^{\circ}$ rotation of the stomach occurs, including the duodenum, pancreas, and dorsal mesentery. The gastric diverticulum is formed by this process of forming a loose muscle layer. This type of diverticulum is present in all layers of the stomach. Second, conversely, false (acquired) diverticulum consists of partial layers of the stomach. This type occurs as a result of secondary conditions such as a malignancy, peptic ulcer disease, pancreatitis, or prior surgery. The false type is most common in adults and is usually located near the gastric antrum. ${ }^{7}$ False diverticula are subdivided into the following groups: (1) the pulsation type, which may arise from conditions associated with increased intraluminal pressure or impaired gastric wall by gastric ulcer or gastric cancer or surgery, or (2) the traction type, which can develop owing to perigastric adhesions and can result from inflammatory lesions of the spleen, gallbladder, pancreas, liver, or peritoneum, or from stomach surgery. ${ }^{8}$ Until recently, surgical management is considered only when medical treatment is refractory or symptomatic, the tumor is large, and complications such as bleeding or perforation are observed. ${ }^{7}$ Since the first successful laparoscopic resection was done for the treatment of a gastric diverticulum in the late 1990s, the safety and feasibility of laparoscopic resection have been shown to be comparable to those of partial gastrectomy. ${ }^{9}$ However, no studies have reported successful treatment of EGC with the endoscopic approach in false gastric diverticulum cases.

Until now, the relation between gastric diverticulum and gastric cancer has not been clearly defined. This coexistence has been reported in a few case reports. ${ }^{10,11}$ In our case, the EGC was type I+IIc, shaped with a central depressed area, and resembled a gastric diverticulum. A previous intra-abdominal operation would contribute to this inverted type of EGC. Precise evaluation of the EGC was impossible in the endoscopic examination because of the lack of mucosa, inflammation, and submucosal infiltration. Also, the comorbidity of the patient and the risk due to the previous surgery indicated that an endoscopic approach was needed. Therefore, ESD was done to resect the false gastric diverticulum that coexisted with the EGC. The main lesion had an invaded submucosal layer (sm1) in the endoscopic examination. For the procedure, marking with argon plasma coagulation was performed first, as usual; then, most of the lesion was dissected with an IT knife. For 
the dissection of a lesion with a diverticular shape, the submucosal layer should be definitively identified and careful dissection is pertinent. For this reason, a hook knife was used to dissect the central area where the fibrosis seemed to be severe in this case. Compared with an IT knife, dual-type and hooktype knives are favored for dissecting fibrotic areas within a narrow field of view.

After 3 months, follow-up EGD and five endoscopic forceps biopsies for the post-ESD scar lesion were performed. There was no evidence of recurrence of EGC in histologic examination. He is scheduled to visit our hospital after 6 months.

ESD has been established as a standard treatment option for EGC with an absolute or extended indication. Most EGC lesions have a typical and easily resectable shape. However, endoscopists are generally reluctant about performing the endoscopic maneuver for EGC with an unusual or difficult-to-resect shape. This is the first report to demonstrate the application of endoscopic resection to an unusually shaped, inverted-type, EGC-forming false gastric diverticulum, and its successful treatment with ESD.

Conflicts of Interest

The authors have no financial conflicts of interest.

\section{REFERENCES}

1. Japanese Gastric Cancer Association. Japanese classification of gastric carcinoma: 3rd English edition. Gastric Cancer 2011;14:101-112.

2. Sano T, Aiko T. New Japanese classifications and treatment guidelines for gastric cancer: revision concepts and major revised points. Gastric Cancer 2011;14:97-100

3. Ono $\mathrm{H}$, Kondo $\mathrm{H}$, Gotoda $\mathrm{T}$, et al. Endoscopic mucosal resection for treatment of early gastric cancer. Gut 2001;48:225-229.

4. Gotoda T. Endoscopic resection of early gastric cancer. Gastric Cancer 2007;10:1-11.

5. Moses WR. Diverticula of the stomach. Arch Surg 1946;52:59-65.

6. Seltzer MH, Koch AW. A huge gastric diverticulum. Am J Dig Dis 1971;16:167-170.

7. Rodeberg DA, Zaheer S, Moir CR, Ishitani MB. Gastric diverticulum: a series of four pediatric patients. J Pediatr Gastroenterol Nutr 2002;34:564-567.

8. Anaise D, Brand DL, Smith NL, Soroff HS. Pitfalls in the diagnosis and treatment of a symptomatic gastric diverticulum. Gastrointest Endosc 1984;30:28-30.

9. Fine A. Laparoscopic resection of a large proximal gastric diverticulum. Gastrointest Endosc 1998;48:93-95.

10. Adachi Y, Mori M, Haraguchi Y, Sugimachi K. Gastric diverticulum invaded by gastric adenocarcinoma. Am J Gastroenterol 1987;82:807.

11. Fork FT, Toth E, Lindstrom C. Early gastric cancer in a fundic diverticulum. Endoscopy 1998;30:S2 